\title{
ANALYTIC MAPPINGS BETWEEN ARBITRARY RIEMANN SURFACES ${ }^{1}$
}

\author{
BY LEO SARIO
}

Communicated by John W. Green, July 10, 1962

1. The problem. L. Ahlfors [1] established the first and second main theorems for analytic mappings from the plane $R$ into a closed Riemann surface $S$. Recently S. Chern [3] extended these theorems to the case where $R$ is a closed Riemann surface less a finite number of points, and $S$ is closed. He also announced a generalization to an $R$ that is obtained from a closed surface by removing a finite number of points and disks. Here we shall consider an arbitrary open surface $R$, of finite or infinite genus, and an arbitrary closed or open $S$.

Chern's elegant method made substantial use of results in related fields: differential geometry, projective geometry, topology, and partial differential equations. Our approach is elementary and purely analytic: the only tools used are the principal functions.

The theorems will be formulated so as to include earlier results. In the classical case of the plane or the disk we obtain a new form of the second main theorem where exceptional intervals are not needed.

2. Singularity function. Let $a_{1}, \cdots, a_{q}, \zeta_{0}, \zeta_{1}$, be points on an arbitrary closed or open Riemann surface $S$ with local variable $\zeta$. Construct the principal function $t_{0}$ with singularities $-c \log \left|\zeta-\zeta_{0}\right|$ and $c \log \left|\zeta-\zeta_{1}\right|, 0<c$ (cf. [2; 4]). Near the ideal boundary choose $t_{0}=L_{0} t_{0}$ where $L_{0}$ is the operator associated with vanishing normal derivative. Normalize $t_{0}$ by $t_{0}(\zeta)+c \log \left|\zeta-\zeta_{0}\right| \rightarrow 0$ as $\zeta \rightarrow \zeta_{0}$, and set $s_{0}=\log \left(1+e^{t_{0}}\right)$.

For $a \neq \zeta_{0}$ let $t=t(\zeta, a)$ be the principal function with a harmonic $t(\zeta, a)+c \log |\zeta-a|$ at $a$. At $\zeta_{0}$ let $t(\zeta, a)-c \log \left|\zeta-\zeta_{0}\right|$ be harmonic and tend to $s_{0}(a)$ as $\zeta \rightarrow \zeta_{0}$. Again choose the boundary behavior $t=L_{0} t$. The function $s(\zeta, a)=s_{0}(\zeta)+t(\zeta, a)$ is symmetric:

$$
s(a, b)=s(b, a) .
$$

Endow $S$ with the conformal metric $\lambda(\zeta)|d \zeta|$ where

$$
\lambda^{2}=\Delta s=\frac{e^{t_{0}}\left|\operatorname{grad} t_{0}\right|^{2}}{\left(1+e^{t_{0}}\right)^{2}} .
$$

The total area $\mu$ of $S$ is $\leqq 2 \pi c$ and the Gaussian curvature is $K=1$.

${ }^{1}$ Sponsored by the U. S. Army Research Office (Durham), Grant DA-ARO(D)31-124-G40, University of California, Los Angeles. 
Let $d \omega(\zeta)$ be the Euclidian area element of $S$ and set $d \mu(\zeta)=\lambda^{2}(\zeta) d \omega(\zeta)$.

3. First main theorem. Consider an arbitrary open Riemann surface $R$ and an analytic mapping $\zeta=f(z)$ of $R$ into $S$. Let $R_{0}$ be a compact bordered subregion [2] of $R$ with border $\beta_{0}$ such that $f(z)$, $z \in \beta_{0}$, is different from $a_{1}, \cdots, a_{q}, \zeta_{0}, \zeta_{1}$, and from the zeros of $\lambda$. Let $\Omega$ be a relatively compact bordered region in $R-\bar{R}_{0}$ with border $\beta_{0}+\beta_{\Omega}$.

Consider the subregion $\Omega_{h}$ of $\Omega$, bordered by $\beta_{0}$ and the level line $\beta_{h}: v=h, 0 \leqq h \leqq k$. Form the harmonic function $u$ on $\bar{\Omega}_{h}$ with $u=0$ on $\beta_{h}, u=h=$ const. $>0$ on $\beta_{0}, \int_{\beta_{0}} d u^{*}=-1$, and set $v=h-u$. For any function $\phi$ in $\Omega$ denote by $n(h, \phi)$ the number of zeros of $\phi$ in $\Omega_{h}$. We set $d \mu_{z}=\left|f^{\prime}(z)\right|^{2} \lambda(f(z)) d w_{z}$, where $d w_{z}$ is the Euclidian area element in $R$, designate symbolically by $n(h, f-a)$ the number of $a$-points of $f$ in $\Omega_{h}$, and introduce:

$$
\begin{aligned}
A(h, a) & =2 \pi c \int_{0}^{h} n(h, f-a) d h, \\
B(h, a) & =\int_{\beta_{h}} s(f(z)) d v^{*} \\
C(h) & =\int_{\Omega_{h}} u(z) d \mu_{z} \\
D(h, a) & =B(0, a)+h B^{\prime}(0, a) .
\end{aligned}
$$

Here $A(h, a)$ serves as the counting function, $B(h, a)$ as the proximity function, and $C(h, a)$ as the characteristic.

Green's formula applied to $u$ and $s$ gives:

First MaIn Theorem. For arbitrary analytic mappings and every $\Omega \subset R$,

$$
A(k, a)+B(k, a)=C(k)+D(k, a) .
$$

The only functions of interest will be those with $k / C(k) \rightarrow 0$ as $\Omega \rightarrow R$, and for such functions $D(k, a)$ is a negligible remainder.

Stokes formula applied to $d s^{*}$ gives

$$
2 \pi c n(h, f-a)+\int_{\beta_{h}-\beta_{0}} d s^{*}=\int_{\Omega_{h}} d \mu_{z},
$$

showing that $C^{\prime}(h)=\int_{\Omega_{h}} d \mu_{z}$.

4. Second main theorem. Had we normalized $\mu$ (instead of $K$ ) to be unity, an integration of (4) with respect to such $d \mu_{0}$ would give 
$\int_{S} B(h, a) d \mu_{0}=O(h)$. Thus the points $a$ that give substantial contributions to the proximity function are exceptional. This suggests a second main theorem even in the present general situation.

For any function $\phi(h, a)$ let $\phi(h)=\sum_{1}^{a} \phi\left(h, a_{i}\right)$. Then

$$
A(h)+B(h)=q C(h)+D(h) .
$$

Let $s_{i}(\zeta)=s\left(\zeta, a_{i}\right), \sigma=\exp \left(\sum_{1}^{a} s_{i} /(1+\epsilon)\right), \epsilon>0$, and

(6)

$$
G(h)=\int_{\beta_{h}} \log \left(\sigma \delta^{2}\right) d v^{*},
$$

$$
H(h)=-2 \int_{\beta_{h}} \log \delta d v^{*},
$$

where $\delta$ is the density induced in the $\left(v+i v^{*}\right)$-plane by $\lambda$,

$$
\delta(z)=\lambda(f(z))\left|f^{\prime}(z)\right||\operatorname{grad} v|^{-1} .
$$

Then $B(h)=(1+\epsilon)(G(h)+H(h))$.

We obtain, by first evaluating $H^{\prime}(h)$ and then integrating:

$$
\begin{aligned}
H(h)= & H(0)+4 \pi\left[-N(h, \lambda)-N\left(h, f^{\prime}\right)+E(h)\right] \\
& +(c-2) \pi\left[N\left(h, \zeta_{0}\right)+N\left(h, \zeta_{1}\right)\right]+2 C(h) .
\end{aligned}
$$

Here $N=\int_{0}^{h} n d h, e$ is the Euler characteristic of $\Omega_{h}$, and $E=\int_{0}^{h} e d h$.

For any function $\phi$ in $(0, k)$ set $\phi_{2}(h)=\int_{0}^{h} \int_{0}^{y} \phi(x) d x d y$. In terms of $I(h)=\int_{\beta_{h}} \sigma \delta^{2} d v^{*}$ we have by the convexity of the logarithm

$$
G_{2}(h)<h^{2} \log I_{2}(h)+O\left(h^{3}\right) .
$$

The distribution $d m=\sigma d \mu_{z}$ gives finite mass $m=\int_{s} d m$ and the integral $M(\zeta)=\int_{S} s(\zeta, a) d m(a)$ converges by (1). Integration of (4) with respect to $d m(a)$ over $S$ gives

$$
2 \pi c I_{2}(h)=m C(h)-M(h)+O(h)
$$

where $M(h)=\int_{\beta_{h}} M(\zeta) d v^{*}$.

On substituting into (5), redenoting $2 \epsilon$ by $\epsilon$, and letting $c=2, h=k$, one obtains the following implicit form of the

Second Main Theorem. For every $\Omega \subset R$, and arbitrary $R$ and $S$,

$$
\begin{aligned}
(q-2-\epsilon) C(k)< & 4 \pi\left[N(k)-N(k, \lambda)-N\left(k, f^{\prime}\right)+E(k)\right] \\
& +G(k)+O(k),
\end{aligned}
$$

where $N(k)=\sum_{1}^{a} N\left(k, f-a_{i}\right)$ and $G(k)$ satisfies (9), (10).

5. Applications. Add to $a_{1} \cdots a_{q}$ points $a_{q+1}, \cdots, a_{q+p}$ among the 
zeros of $\lambda$ including at least all those covered by $f(\Omega)$. Then $\sum_{a+1}^{a+p} N\left(k, a_{i}\right)-N(k, \lambda)=0$ and

$$
\begin{aligned}
(q+p-2-\epsilon) C(k) & <4 \pi\left[\sum_{1}^{q} N\left(k, a_{i}\right)-N\left(k, f^{\prime}\right)+E(k)\right] \\
& +G(k)+O(k) .
\end{aligned}
$$

Instead of giving an explicit estimate, with exceptional intervals, for $G$ from $G_{2}$ it is simpler to replace $B$ by the integral $B_{2}$ of the "areal" proximity $\int_{0}^{h} B d h$. The scarcity of points $a$ contributing to $B_{2}$ remains valid when $A, C, D$ in (5) are replaced by $A_{2}, C_{2}, D_{2}$. In (12) $O(k)$ is replaced by $O\left(k^{3}\right)$, the subindex " 2 " is attached to all other capital letters, and $G_{2}(k)$ is directly given by (9), (10).

If $S$ is closed, then $M(\zeta)$ is bounded on $S$ and $M(k)=O(1)$ in $k=k(\Omega), \Omega \rightarrow R$. There are $p=2 g$ zeros of $\lambda$, and $p-2$ is the Euler characteristic $e_{S}$ of $S$. We have the following

COROLlARY. In an analytic mapping of an arbitrary open $R$ into a closed $S$,

$$
\left(q+e_{S}-\epsilon\right) C_{2}(k)<4 \pi\left[N_{2}(k)-N_{2}\left(k, f^{\prime}\right)+E_{2}(k)\right]+G_{2}(k),
$$

where

$$
G_{2}(k)=O\left(k^{2} \log C(k)+k^{3}\right) .
$$

We choose the defect

$$
\delta(a)=1-\limsup _{\Omega \rightarrow R} \frac{4 \pi N_{2}(k)}{C_{2}(k)}
$$

and set

$$
\eta=\limsup _{\Omega \rightarrow R} \frac{4 \pi E_{2}(k)}{C_{2}(k)} .
$$

For functions with $G_{2}(k) / C_{2}(k) \rightarrow 0$ we obtain the following.

Defect Relation.

$$
\sum \delta\left(a_{i}\right) \leqq \eta-e_{S}
$$

In particular, there can be at most $\eta-e_{S}$ Picard values.

Functions for which $C_{2}$ grows more rapidly than $E_{2}$ cannot give a mapping into an $S$ with $g>1$. For an $R$ that is a closed surface punctured at a finite number of points this is Chern's theorem.

In the special case where $R$ has a capacity function $p_{\beta}$ with compact level lines, this can be taken for $v$, and the directed limits for $\Omega \rightarrow R$ 
replaced by limits as $k \rightarrow \sup _{R} p_{\beta}$. By l'Hopital's rule the quantities $C_{2}, N_{2}, E_{2}$ in (15), (16) can then be replaced by $C, N, E$, and we find the usual forms.

For meromorphic functions in the plane or the disk take $v=(1 / 2 \pi) \log r$. Then (13) is a new form of the second main theorem that holds without exceptional intervals.

\section{BiBLIOGRAPHY}

1. L. Ahlfors, Über die Anwendung differentialgeometrischer Methoden zur Untersuchung von Überlagerungsflächen, Acta Soc. Sci. Fenn. Nova Series A. II. No. 6 (1937), 1-17.

2. L. Ahlfors and L. Sario, Riemann surfaces, Princeton Univ. Press, Princeton, N. J., 1960.

3. S. Chern, Complex analytic mappings of Riemann surfaces. I, Amer. J. Math. 82 (1960), 323-337.

4. L. Sario, $A$ linear operator method on arbitrary Riemann surfaces, Trans. Amer. Math. Soc. 72 (1952), 281-295.

University of California, Los Angeles 